\title{
INTER-CELL INTERFERENCE ANALYSIS FOR OFDM/FBMC SYSTEMS
}

\author{
Y. Medjahdi*, M. Terré*, D. Le Ruyet*, D. Roviras*, J.A. Nossek ${ }^{\dagger}$ and L. Baltar ${ }^{\dagger}$ \\ * Electronics and Communications Laboratory, CNAM, Paris, France. \\ 'Institute for Circuit Theory and Signal Processing \\ Technische Universität München, Munich, Germany
}

\begin{abstract}
Multicarrier communication technologies are promising candidates for $4 \mathrm{G}$ wireless access systems. In this paper, we focus on the downlink of multicellular networks and we investigate the influence of the inter-cell interference in an unsynchronized frequency division duplex (FDD) context with a frequency resuse of 1 . We compare the conventional orthogonal frequency division multiplexing with cyclic prefix modulation (CP-OFDM) and the filter bank based multi-carrier modulation (FBMC). Two tables modeling the mean interference power are given and show that, in FBMC case, the interference is more localized than in OFDM case. Finaly, these tables are used to evaluate performance in terms of average capacity in FBMC multi-cell networks compared to CP-OFDM ones.
\end{abstract}

Index Terms - CP-OFDM, FBMC, inter-cell interference, mean interference power tables

\section{INTRODUCTION}

Future communication systems are expected to support multimedia rate-demanding services such as audio, video and internet applications and to provide much higher data rates than today's mobile communication systems (3G). OFDM is one of the leading candidates for providing high data rates due to its robustness to multipath effects and efficient implementation using FFT.

In some cases (cognitive radio, non cooperative base stations,...), it is not possible to synchronize the different base stations (BS). Consequently, it is relevant to evaluate the impact of this non synchronization in the time domain on the system's performance.

However, to provide high data rates, it is necessary to use large bandwidths and aggressive frequency-reuse plans that will greatly increase the interference in nonsynchronized cellular communications. In OFDM-based cellular systems, several works have been reported on the inter-cell interference caused by the temporal desynchronization [1,2]. A previous study on modeling interferences in unsynchronized cellular network was also proposed in [3].

This work was supported in part by the European Comission under Project PHYDYAS (FP7-ICT-2007-1-211287)
Recently, a number of papers have focused on a new alternative called Filter bank based multicarrier system (FBMC) which can offer a number of advantages over CPOFDM system such as the improved spectral efficiency by not using a redundant $\mathrm{CP}$ and by having much better control of out-of-band emission due to the time-frequency localized shaping pulses $[4,5]$.

In this paper, we compare OFDM and FBMC in the downlink of a multicellular network. We focus on the impact of inter-cell interference in an unsynchronized FDD context. It means that the other BS(s) are the only interferers (other-entity interference). We will also assume a frequency reuse factor of one, meaning that all the cells present in the network use the same frequency bands.

We assume a perfect frequency and time synchronization between the user of interest and its own BS. Hence, the interference will only come from the other cells. Our aim is to analyse the inter-cell interference power in FBMC and OFDM systems and to lead a performance comparison of these two modulation techniques.

In our analysis, frequency offset will be disregarded. Nevertheless, as the two cells are not time synchronized, we will be interested in the timing offset $\tau$ and phase offset $\varphi$ between the user of interest and the BS of the other cell.

The outline of this paper is organized as follows: Section 2 describes the system model together with a general mathematical model developed to evaluate the amount of interference in both systems due to timing offsets and phase offsets. In Section 3, the signal-to-interference ratio (SIR) as well as the capacity are analysed to compare the performance of the two systems in consideration. Finally, conclusions are summarized in Section 4.

\section{SYSTEM MODEL}

To take into account the detrimental effects of interference caused by adjacent cells, we refer to a two-cell layout with one user located at the border of the cell and $\mathrm{BS}(\mathrm{s})$ situated in the center of each hexagonal cell.

For the sequel of the paper, a subcarrier is called "frequency slot" and the OFDM symbol duration is called "time slot". 
In the OFDM case, we note that the inter-cell interference comes from two consecutive time slots and from a high number of frequency slots used in neighbouring cells. We can then define a set $\Omega_{\text {OFD }}$ of time/frequency slots of the other cell that are interfering with the analyzed time/frequency slot of the cell of interest, so called "victim slot".

\subsection{Derivation of interferences for CP-OFDM system}

Consider the transmission of a single complex symbol $x=[a+j b]$ on the $k$-th frequency slot, from the interfering $\mathrm{BS}$ which is defined by

$$
s(t)=x g(t-\tau) e^{j \frac{2 \pi}{T} k(t-\tau)+j \varphi}
$$

The transmit pulse shape $g(t)$ and the receiver filter impulse response $f(t)$ are expressed as follows

$$
\begin{gathered}
g(t)=\left\{\begin{array}{c}
1 / \sqrt{T}, t \in[0, T+\Delta] \\
0 \text { elsewhere }
\end{array}\right. \\
f(t)=\left\{\begin{array}{c}
1 / \sqrt{T}, t \in[0, T] \\
0 \text { elsewhere }
\end{array}\right.
\end{gathered}
$$

where $\Delta$ is the CP duration.

The $k$ '-th output of the receiver filter in the victim slot for a timing offset $\tau$ is given by

$$
\begin{aligned}
y_{k^{\prime}}(\tau, \varphi) & =\left.\left[s(t) e^{-j \frac{2 \pi}{T} k^{\prime} t}\right] * f(t)\right|_{t=T+\Delta} \\
& =x e^{-j \frac{2 \pi}{T} k \tau+j \varphi} \int_{-\infty}^{+\infty} g\left(t^{\prime}-\tau\right) f\left(T+\Delta-t^{\prime}\right) e^{j \frac{2 \pi}{T} l t^{\prime}} d t^{\prime}
\end{aligned}
$$

where $k$ is the interfering frequency slot while $k$ ' is the victim frequency slot. $l=k-k$ ' denotes the offset between them.

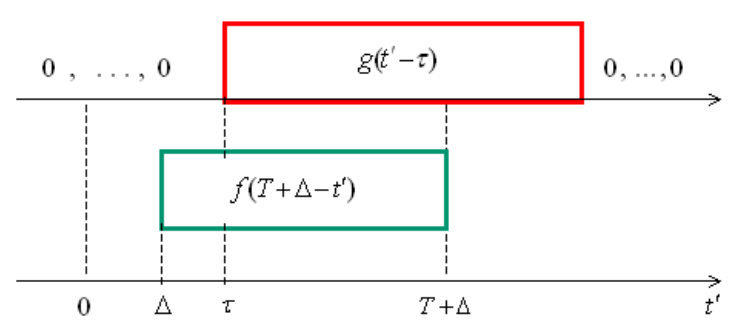

Figure 1: Respective positions of transmitter and receiver pulses.

In general case, we see that the product $g\left(t^{\prime}-\tau\right) f\left(T+\Delta-t^{\prime}\right)$ and the value of $\tau$ determine the limits of the integral appearing in (4), we have then three cases to be analyzed in order to calculate $y(\tau)$

Case 1: $0<\tau<\Delta$ (timing offset $\tau$ absorbed by the CP)

$$
y_{k^{\prime}}(\tau, \varphi)=x e^{-j \frac{2 \pi}{T} k \tau+j \varphi} \int_{\Delta}^{T+\Delta} e^{j \frac{2 \pi}{T} l t^{\prime}} d t^{\prime}=x e^{-j \frac{2 \pi}{T} k \tau+j \varphi} \delta(l)
$$

Case 2: $\Delta<\tau<T+\Delta($ see Figure 1$)$

$$
\begin{aligned}
& y_{k^{\prime}}(\tau, \varphi)=x e^{-j \frac{2 \pi}{T} k \tau+j \varphi} \int_{\tau}^{T+\Delta} e^{j \frac{2 \pi}{T} l t^{\prime}} d t^{\prime} \\
&=x e^{-j \frac{2 \pi}{T}\left[k \tau+l\left(\frac{\tau+\Delta}{2}\right)\right]+j \varphi} \frac{\sin (\pi l(\Delta-\tau) / T)}{\pi l}
\end{aligned}
$$

Case 3: $T+\Delta<\tau<T+2 \Delta$

$$
y_{k^{\prime}}(\tau, \varphi)=0
$$

The corresponding interference power is

$I(\tau, \varphi, l)=\left|y_{k^{\prime}}(\tau, \varphi)\right|^{2}= \begin{cases}\delta(l) & \tau \in[0, \Delta] \\ \mid \frac{\sin (\pi l(\Delta-\tau) / T)}{\pi l} & \tau \in[\Delta, T+\Delta] \\ 0 & \tau \in[T+\Delta, T+2 \Delta]\end{cases}$

according to (8), we find that the interference power does not depend on the phase offset $\varphi$.

For computing the mean interference power, we assume a uniformly distributed offset $\tau$. We have chosen the interval $\tau \in[\Delta / 2, \mathrm{~T}+3 \Delta / 2]$ to obtain a symmetry between the interference power coming from two consecutive time slots. Then, we have

$$
\bar{I}(l)=\frac{1}{T+\Delta} \int_{\Delta / 2}^{T+3 \Delta / 2} I(\tau, l) d \tau
$$

For $l=0$, we get

$$
\bar{I}(0)=\frac{2 T+3 \Delta}{6(T+\Delta)}
$$

and for $l \neq 0$

$$
\bar{I}(l)=\frac{T}{2(T+\Delta)} \frac{1}{(\pi l)^{2}}
$$

As expected, the interference comes from a large number of frequency slots and only from two successive time slots.

It should be noted that, the choice of another integration interval could give another table which is not always symmetric. Moreover, for some intervals, the average power table could be spreaded over 3 time slots. However, this case is not relevant since, for a given timing offset $\tau$, the interference comes only from 2 consecutive time slots. 


\subsection{Derivation of interferences for FBMC system}

In a FBMC system, we have also a multicarrier transmission. In order to have a better frequency localization, we can use different transmit pulses (non rectangular) that are more localised in time-frequency domain. Moreover, the lack of the $\mathrm{CP}$ will improve spectral efficiency. To maintain the orthogonality between subcarriers, an offset QAM transmission has to be adopted instead of conventional QAM $[4,5]$. The OQAM technique is based on the offset $T / 2$ introduced between the real part and the imaginary part of each QAM symbol.

Then, the transmit signal is given by

$$
\begin{aligned}
s(t)= & {[a[n] g(t-n T-\tau)} \\
& +j b[n] g(t-n T-\tau-T / 2)] e^{j\left(\frac{2 \pi}{T}(t-\tau)+\frac{\pi}{2}\right) k+j \varphi}
\end{aligned}
$$

$\tau$ denotes the timing offset between the victim user and the other BS.

The $k$ '-th output can thus be expressed

$$
\begin{gathered}
y_{k^{\prime}}(t, \tau, \varphi)=\left(s(t) \cdot e^{-j\left(\frac{2 \pi}{T} t+\frac{\pi}{2}\right) k^{\prime}}\right) * f(t) \\
=e^{-\frac{j \pi}{T} k t+j \varphi}\left\{a[l] \int_{-\infty}^{\infty} g(t-l T-\tau-\alpha) f(\alpha)\right. \\
e^{j\left(\frac{2 \pi}{T}(t-\alpha)+\frac{\pi}{2}\right)\left(k-k^{\prime}\right)} d \alpha \\
+b[l] \int_{-\infty}^{\infty} g\left(t-\left(l+\frac{1}{2}\right) T-\tau-\alpha\right) f(\alpha) \\
\left.e^{j\left(\frac{2 \pi}{T}(t-\alpha)+\frac{\pi}{2}\right)\left(k-k^{\prime}\right)} d \alpha\right\}
\end{gathered}
$$

$g(t)$ is the real-valued symmetric pulse response of the prototype filter and $f(t)$ is matched to $g(t)$, i.e. $g(t)=f(t)=f(-t)$.

After the decision, the interference power can be written as

$$
I_{n, k^{\prime}}(\tau, \varphi)=\left|\operatorname{Re}\left[y_{k^{\prime}}(t)\right]_{t=n T}+j \operatorname{Im}\left[y_{k^{\prime}}(t)\right]_{t=(n+1 / 2) T}\right|^{2}
$$

In this case, the interference does not only depend on the timing offset $\tau$ but also on the phase offset $\varphi$.

The corresponding mean interference power is calculated for an uniformly distributed timing offser $\tau \in[T / 2,3 T / 2]$ and also for an uniformly distributed phase offset $\varphi \in[0,2 \pi]$

$$
\bar{I}_{k^{\prime}}(n)=\frac{1}{2 \pi T} \iint_{\tau, \varphi} I_{k^{\prime}}(n, \tau, \varphi) d \tau d \varphi
$$

In our analysis, we have considered the PHYDYAS NPR (nearly perfect reconstruction) prototype filter using the frequency sampling technique [6]. For this prototype filter, the length of the impulse response of the filter is linked to the time slot duration $T$ by $L=4 T$. Compared to OFDM case, the interference comes from $\mathrm{K}$ time slots where $\mathrm{K}$ is function of the prototype filter length $L$.

However, we give only simulated results in the next subsection for expression (15).

\subsection{Mean interference power tables}

For CP-OFDM system, theoretical results for $\Delta=\mathrm{T} / 8$, are given in Table 1. It corresponds to a transmitted power that equals 1 with channel gains of 1 between the two BSs and the victim user. As expected, it appears that the interference power is spread over a high number of frequency slots and only two successive time slots.

On the other hand, Table 2 shows the interference distribution for FBMC system. In this case, the interference is more localized in frequency but it comes from a number of time slots which depends on the length of prototype filter.

It should be noted that for the two tables, we considered only the main interfering slots, with an interference power greater than $10^{-3}$. We obtain then, 15 interfering slots for the FBMC case, and 30 slots for the OFDM case.

\begin{tabular}{|c|c|c|}
\hline$f^{t}$ & $n$ & $n+1$ \\
\hline$k+7$ & $9.19 \mathrm{E}-04$ & $9.19 \mathrm{E}-04$ \\
\hline$k+6$ & $1.25 \mathrm{E}-03$ & $1.25 \mathrm{E}-03$ \\
\hline$k+5$ & $1.80 \mathrm{E}-03$ & $1.80 \mathrm{E}-03$ \\
\hline$k+4$ & $2.81 \mathrm{E}-03$ & $2.81 \mathrm{E}-03$ \\
\hline$k+3$ & $5.00 \mathrm{E}-03$ & $5.00 \mathrm{E}-03$ \\
\hline$k+2$ & $1.13 \mathrm{E}-02$ & $1.13 \mathrm{E}-02$ \\
\hline$k+1$ & $4.50 \mathrm{E}-02$ & $4.50 \mathrm{E}-02$ \\
\hline$k$ & $3.52 \mathrm{E}-01$ & $3.52 \mathrm{E}-01$ \\
\hline$k-1$ & $4.50 \mathrm{E}-02$ & $4.50 \mathrm{E}-02$ \\
\hline$k-2$ & $1.13 \mathrm{E}-02$ & $1.13 \mathrm{E}-02$ \\
\hline$k-3$ & $5.00 \mathrm{E}-03$ & $5.00 \mathrm{E}-03$ \\
\hline$k-4$ & $2.81 \mathrm{E}-03$ & $2.81 \mathrm{E}-03$ \\
\hline$k-5$ & $1.80 \mathrm{E}-03$ & $1.80 \mathrm{E}-03$ \\
\hline$k-6$ & $1.25 \mathrm{E}-03$ & $1.25 \mathrm{E}-03$ \\
\hline$k-7$ & $9.19 \mathrm{E}-04$ & $9.19 \mathrm{E}-04$ \\
\hline
\end{tabular}

Table 1: Theoretical mean interference power table $T^{O F D M}$.

\begin{tabular}{|c|c|c|c|c|c|}
\hline$f$ & $n-2$ & $n-1$ & $n$ & $n+1$ & $n+2$ \\
\hline$k-1$ & $1.08 \mathrm{E}-03$ & $1.99 \mathrm{E}-02$ & $4.60 \mathrm{E}-02$ & $1.99 \mathrm{E}-02$ & $1.08 \mathrm{E}-03$ \\
\hline$k$ & $1.05 \mathrm{E}-03$ & $1.26 \mathrm{E}-01$ & $5.69 \mathrm{E}-01$ & $1.26 \mathrm{E}-01$ & $1.05 \mathrm{E}-03$ \\
\hline$k+1$ & $1.08 \mathrm{E}-03$ & $1.99 \mathrm{E}-02$ & $4.60 \mathrm{E}-02$ & $1.99 \mathrm{E}-02$ & $1.08 \mathrm{E}-03$ \\
\hline
\end{tabular}

Table 2: Simulated mean interference power table $T^{F B M C}$.

These two tables give us a useful tool for inter-cell interferences computation. Thus, performance analysis in a more realistic multi-cellular scenario can easily be 
extrapolated without performing a completed physical layer simulation.

\section{SIR AND CAPACITY CALCULATION}

\subsection{SIR and mean interference tables}

The interference tables can easily be used for the derivation of the mean interference power due to a set of active slots in the interfering cell.

Let us consider a simple case where two slots $(p, q)$ and $(u, v)$ of the interfering cell are actives, the other slots being unactives. These two slots interfere with the $(n, k)$ victim slot of the analyzed cell.

We can then obtain the instantaneous interference (complex value), for a particular value of $\tau$ (time delay representing the non synchronization of the two cells).

We have then

$$
y_{n, k}(\tau)=g_{q} c_{p, q}(\tau) s_{p, q}+g_{v} c_{u, v}(\tau) s_{u, v}
$$

where

- $y_{n, k}(\tau)$ represents the complex interference value received by the victim $\operatorname{slot}(n, k)$.

- $\quad g_{p}\left(\right.$ resp. $\left.g_{q}\right)$ is the channel gain at frequency $p$ (resp. $q$ ), between the other BS and the victim.

- $c_{p, q}(\tau)$ is the complex amplitude coupling coefficient between the active $\operatorname{slot}(p, q)$ and the $\operatorname{victim} \operatorname{slot}(n, k)$.

- $s_{p, q}$ represents the modulation symbol transmitted on slot $(p, q)$ in the interfering Cell.

Evaluating the mean value of the interference power, we have

$$
E\left[I_{n, k}\right]=E\left|g_{q} c_{p, q}(\tau) s_{p, q}+g_{v} c_{u, v}(\tau) s_{u, v}\right|^{2}
$$

The statistical expectation $E[$.$] is computed over$

- all channel gains $\left(g_{p}\right)$

- all time delays $(\tau)$

- all communication symbols $\left(s_{p, q}\right)$

As $g_{p}, c_{p, q}(\tau)$ and $s_{p, q}$ are independent variables, the expectation can be simplified.

Moreover, as communication symbols are zero mean uncorrelated variables, we have:

$$
\begin{aligned}
E\left[I_{n, k}\right]= & E_{\{g\}}\left|g_{q}\right|^{2} E_{\{\tau\}}\left|c_{p, q}(\tau)\right|^{2} E_{\{s\}}\left|s_{p, q}\right|^{2} \\
& +E_{\{g\}}\left|g_{v}\right|^{2} E_{\{\tau\}}\left|c_{u, v}(\tau)\right|^{2} E_{\{s\}}\left|s_{u, v}\right|^{2}
\end{aligned}
$$

Let's consider now

$$
y_{n, k}=\sum_{(p, q) \in \Omega_{a}} g_{q} c_{p, q}(\tau) s_{p, q}
$$

where $\Omega_{a}$ denotes the set of active slots.

Using (17) we have

$$
E\left[I_{n, k}\right]=\sum_{(p, q) \in \Omega_{a}} E_{\{g\}}\left|g_{q}\right|^{2} E_{\{\tau\}}\left|c_{p, q}(\tau)\right|^{2} E_{\{s\}}\left|s_{p, q}\right|^{2}
$$

where

- $E_{\{g\}}\left|g_{q}\right|^{2}$ is the mean square modulus of the channel gain.

- $E_{\{\tau\}}\left|c_{p, q}(\tau)\right|^{2}=T_{p, q}$ is directly given by the interference table.

- $E_{\{s\}}\left|s_{p, q}\right|^{2}=p_{s}$ is the mean power of the transmitted communication symbol.

Finally, we obtain

$$
E\left[I_{n, k}\right]=p_{s}\left(\sum_{(p, q) \in \Omega_{a}} E_{\{g\}}\left|g_{q}\right|^{2} T_{p, q}\right)
$$

If we consider $E_{\{g\}}\left|g_{j}\right|^{2}=\bar{G}_{2}$, we obtain

$$
E\left[I_{n, k}\right]=\bar{G}_{2} p_{s} \sum_{(p, q) \in \Omega_{a}} T_{p, q}
$$

If we consider that the mean useful received power (from the base station of interest) is equal to $\bar{G}_{1} p_{s}$, we have the following expression for the mean Signal to Interference power Ratio $(S I R)$

$$
S I R=\frac{\bar{G}_{1}}{\bar{G}_{2} \sum_{(p, q) \in \Omega_{a}} T_{p, q}}
$$

\subsection{Interference probability}

Power interference tables introduced in (Section 2) can be used for estimating the probability density function of the interference power. If we introduce the probability $p$ representing the probability of having an active transmission in a time/frequency slot, then it appears that we can calculate the probability of having $k$ active time/frequency slots in the $\Omega_{\text {OFDM }}\left(\right.$ resp. $\left.\Omega_{F B M C}\right)$ set

$$
P_{k}=C_{N}^{k} p^{k}(1-p)^{N-k}
$$

where $N$ denotes $\operatorname{Card}\left(\Omega_{\text {OFDM }}\right)$ (resp. $\left.\operatorname{Card}\left(\Omega_{F B M C}\right)\right)$ and $C_{N}^{k}=N ! / k !(N-k) !$.

In the following paragraph, we consider the capacity of each system to compare the performance of the two systems. So, we will be interested in the average capacity of each 
system that can be calculated for a fixed value of traffic load $p$.

For $k$ active slots $k=0, \ldots, N$,we have $C_{N}^{k}$ possibilities, and for each possibility we can obtain the corresponding SIR. The corresponding capacity can be obtained as follows

$$
\text { cap }_{\text {inst }}=\log _{2}(1+S I N R),[\text { bit/s/Hz] }
$$

According to (23) and (24), we obtain the average capacity for a traffic load $(p)$

$$
\operatorname{cap}_{\text {average }}(p)=\sum_{k=0, \ldots, N} P_{k} c a p_{\text {inst }}\left(P_{k}\right)
$$

Figure 2 shows the average capacity of the two systems for $S N R=20 d B$, the curve is obtained by Monte-Carlo simulation.

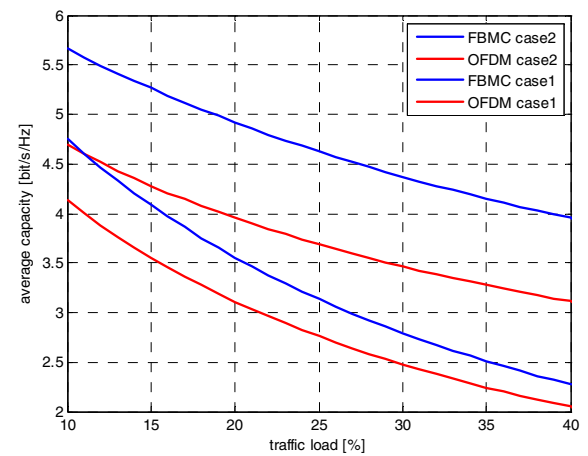

Figure 2: Average capacity in FBMC and OFDM systems.

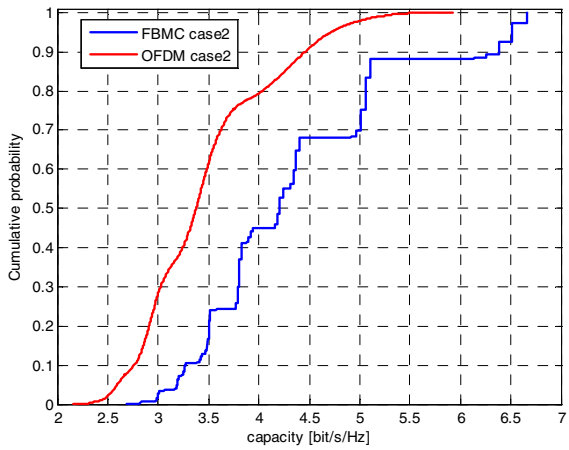

Figure 3: Cumulative probabilty of capacity for a load traffic $p=30 \%, S N R=20 \mathrm{~dB}$.

In the following analysis, we do not consider the path loss between the BS and the user. We assume that the channel gains equal to 1 .

We consider two cases, in the first one (case.1), all frequency slots including the frequency slot of the victim user are used by the interfering BS. This case presents a non-realistic model because the interference is caused by whole bandwidth. Although, the FBMC presents an advantage over the CP-OFDM (improvement of 10\% for a traffic load $p=30 \%$ )
The case. 2 was considered under the assumption that the other BS allocates all frequency slots except the one of the victim user. Comparing to OFDM case2, the average capacity for a traffic load $p=30 \%$ is increased by more than $25 \%$ in the FBMC case 2 .

In figure 3 comparing the two curves, we see that FBMC presents performance lightly better than OFDM system. The $\mathrm{CDF}$ curve of FBMC is not smooth due to the discrete distribution of the mean power interference.

\section{CONCLUSION}

In this paper, the inter-cell interference due to timing offset and phase offset in OFDM and FBMC systems has been analyzed. We have presented the mean interference power tables. These tables give a clear model on the intercell interference which can be used to analyse more realistic multi-cell scenarios without performing a completed physical layer simulation. In the CP-OFDM system, interference comes from many frequency slots and only two consecutive time slots. On the other hand, the interference is more frequency localized because only immediate adjacent subcarriers are causing interference but it is spread out over several symbols in time depending on the prototype filter length. A performance assessment has shown that, compared to CP-OFDM, the FBMC can offer a higher capacity. Therefore interference coordination should be much easier in FBMC context. This will be exploited in future investigations.

\section{REFERENCES}

[1] J-W. Lee, J. Moon, and Y-H. Lee, "Other-Cell Interference Reducing Resource Allocation in OFDM-Based Asynchronous Cellular Systems," EURASIP Journal on Wireless Communications and Networking, vol 2008, Article ID 378097, 2008.

[2] H. Son, S. Lee and G-T. Gil, "The MAI mitigation scheme for OFDMbased Asynchronous Networks over Multi-Cell Environments," IEEE 67th Vehicular Technology Conference (VTC2008-Spring), pp 2331-2335, Marina Bay, Singapore, May 2008.

[3] G. Auer, A. Dammann, S. Sand, and S. Kaiser, "On modelling cellular interference for multi-carrier based communication systems including a synchronization offset," In 6th International Symposium on Wireless Personal Multimedia Communications (WPMC 2003), pp 290-294, Yokosuka, Japan, Oct.2003.

[4] P. Siohan, C. Siclet and N. Lacaille, "Analysis and Design of OFDM/OQAM Systems Based on Filter bank Theory," IEEE Trans. on Signal Proc., vol. 50, no. 5, pp. 1170-1183, May 2002.

[5] H.Boelcskei, "Orthogonal frequency division multiplexing based on offset QAM," in Advances in Gabor analysis. Birkhuser, 2003.

[6] M.Bellanger, "Filter banks and OFDM/OQAM for high throughput wireless LAN," 3rd International Symposium on Communications, Control and Signal Processing, 2008.ISCCSP 2008, pp 758 - 761,Malta, Mar 2008. 